\title{
Variable Speed Limit Strategies Based on the Macro Hierarchical Control Traffic Flow Model
}

\author{
Shubin Li $\mathbb{D},,^{1,2}$ Tao Wang $\mathbb{D},{ }^{3}$ Hualing Ren $\mathbb{D}^{4},{ }^{4}$ Baiying Shi $\mathbb{D}^{2},{ }^{2}$ Xiangke Kong $\mathbb{D}^{2}$ \\ Jianyong Chai $\mathbb{D},{ }^{5}$ and Xuejuan Wang $\mathbb{D}^{6}$ \\ ${ }^{1}$ Department of Traffic Management Engineering, Shandong Police College, Jinan 250014, China \\ ${ }^{2}$ Department of Traffic Engineering, Shandong Jianzhu University, Jinan 250101, China \\ ${ }^{3}$ Department of Automation and Electronic Engineering, Qingdao University of Science and Technology, Qingdao 266061, China \\ ${ }^{4}$ MOE Key Laboratory for Urban Transportation Complex Systems Theory and Technology, Beijing Jiaotong University, \\ Beijing 100044, China \\ ${ }^{5}$ Shandong High-Speed Information Group Co., Ltd, Jinan 250013, China \\ ${ }^{6}$ Department of Rail Transportation, Shandong Jiaotong University, Jinan 250357, China
}

Correspondence should be addressed to Shubin Li; li_shu_bin@163.com, Tao Wang; wangtao@qust.edu.cn, Hualing Ren; hlren@ bjtu.edu.cn, Baiying Shi; shibaiying@sdjzu.edu.cn, and Xiangke Kong; 15621852679@163.com

Received 26 March 2021; Revised 6 June 2021; Accepted 15 June 2021; Published 24 June 2021

Academic Editor: Yong Wang

Copyright (c) 2021 Shubin Li et al. This is an open access article distributed under the Creative Commons Attribution License, which permits unrestricted use, distribution, and reproduction in any medium, provided the original work is properly cited.

\begin{abstract}
The superior traffic control system can promote the efficiency of mainstream expressway. As the effective method to smooth traffic system, the variable speed limits (VSL) strategies are discussed in an expressway traffic network. The dynamic OD estimation model is used to produce the real traffic information, which is loaded to the traffic network. Then, the prediction information of traffic variables and the VSL strategy are introduced to macro hierarchical control traffic flow model. A solution algorithm is further developed to find the optimal parameters of VSL by minimizing the total travel time and delay. The simulation results show that the proposed strategy perfects well, the traffic congestion is effectively alleviated, and the traffic efficiency of the road section is significantly improved. This framework can be adopted by transit managers for traffic efficiency.
\end{abstract}

\section{Introduction}

The expressway is a modern transportation infrastructure which has its specific characteristics, such as high flow and high speed. Building expressway becomes a major project to relieve traffic congestion and traffic delay. This project could improve regional development and promote industrial restructuring and social progress. However, it also attracts more traffic demand for its high efficiency. Then, the problem of traffic congestion and traffic safety occurred inevitably. According to the survey results of Baidu Map in 2020, the degree of highway congestion in China has increased significantly, and the number of traffic jams has also increased. This phenomenon is especially obvious during holidays and peak travel periods. Therefore, it is urgent to take effective control measures to address these problems.
At present, the static speed limit sign is widely used in the speed limit control of expressways, but the determination of the static speed limit value is often based on ideal traffic conditions, and the influence of variable factors such as road traffic flow and weather conditions on the safe driving speed of vehicles is seldom considered. In order to overcome the deficiency of static speed limit control, the dynamic traffic control method represented by variable speed limit (VSL) has gradually become a control strategy considered by scholars and traffic control departments. A large number of studies have found that a smooth traffic flow can effectively improve the safety of vehicles and the efficiency of road sections. Variable speed limit can consider the influence of the change of traffic environment on safe speed more comprehensively and carry out real-time control by adjusting the speed limit value, which is of great significance 
to improve the traffic safety and operation efficiency of expressways.

The dynamic traffic control is an effective method to relieve traffic congestion, which is mainly divided into two types: global control strategy and local control strategy. Both of them play essential roles in relieving the congestion of expressway and ensuring the travel safety [1-3]. Compared with local control, the global control measures have many advantages from the network level, such as global logistics network optimization [4] and global state space-time network model $[5,6]$, take the solution of local traffic congestion as an example for analysis. The local control mitigates the congestion of a specific location on the traffic network. However, this good result comes at the cost of worse congestion at other links in the traffic network, while the control strategy on the network level could avoid this deficiency and reduce the adverse impact of local control on the traffic states of other links. Local controllers only use part of the traffic information, while in the controller on the traffic network level, the real origin-destination (OD) is crucial. The predictions of traffic flow will be easily applicable to control on the network level when the dynamic OD is available.

Therefore, how to implement variable speed limit control strategy in expressway from the perspective of global control becomes the focus of this paper. In addition, in order to adapt the control strategy to the future traffic situation, this paper studies the combination of traffic volume prediction and control of expressway trunk line with variable speed restriction. The paper is divided into eight sections. Section 1 introduces the background and research significance of the problem. Section 2 reviews previous research. Section 3 states the traffic demand estimation and prediction. Section 4 gives the proposed control model and the improved macroscopic traffic flow model. Section 5 presents the simulation method and algorithm. Section 6 shows the numerical examples.Section 7 compares the research results of this model with other models. Section 8 draws the conclusion.

\section{Literature Review}

In variable speed limits research, scholars have done a lot of excellent research. Lin et al. [7] proposed VSL algorithms which were combined with ramp control (RM) with the aim of improving traffic efficiency. Hegyi et al. [8] used the variable speed limit strategy to study the traffic wave and it can compress the wave. Bertini et al. [9] studied the variable speed limit and traveler information system provided by overhead dynamic information signs in order to improve the understanding of how these systems affect driver behavior and bottleneck formation and location. Abdel et al. [10] analyzed the relationship between the implementation of variable speed limit and the possibility of vehicle collision under different traffic conditions and gave some suggestions on the implementation of variable speed limit. Yao proposed a trajectory smoothing method based on individual variable speed limits with location optimization (IVSL-LC) and verified that the method can improve traffic efficiency and fuel consumption [11]. Ellen proposed a variable speed limit system based on connected vehicles and verified that, in most cases, the VSL system can improve traffic efficiency through microsimulation software [12]. In addition, some other applications and studies have also demonstrated the important role of variable speed limit in the safety and efficiency of expressways [13-16].

Besides the direct application of VSL on traffic system, it is also introduced into micro- and mesotraffic flow model $[17,18]$ and other optimization models [19-22]. Abdel et al. used microscopic simulation models to analyze the effects of different speed limit strategies on alleviating and improving road safety [17]. Inanjko established a microscopic simulation framework to analyze the layout and application scenarios of the VSL controller using travel time and vehicle emissions as indicators [18]. Fang proposed a VSL control algorithm based on the model predictive control (MPC) framework. The improved model reduces the speed prediction accuracy error and improves the performance of the system [19]. Alasiri used microscopic simulation software to combine the cell transmission model (CTM) and variable speed limit strategy to verify the effectiveness of the robust VSL controller [20]. Qu used the proposed single-lane cellular automaton model to simulate the traffic flow characteristics under VSL control. The simulation results are consistent with the actual situation, which verifies the validity of the model [21]. Li et al. combined the variable speed limit strategy with the cellular conveyor model to optimize the traffic flow of expressways [22].

The good performance of VSL combined with traffic flow model is demonstrated in their work [23-26], but there are still some unresolved problems need to be addressed. The gaps need to be filled are listed as follows: (1) the research area is small, and a large number of studies mainly focus on the ramp area of expressways, lacking the research on long-distance trunk lines; (2) the simulation data are mainly based on historical traffic volume, and lack of prediction of future traffic volume changes, resulting in low reference of simulation results. At present, the research on traffic flow modeling and prediction has also made great progress $[27,28]$, but it is rarely applied to VSL; (3) a large number of studies start from the microperspective, but there is lack of analysis from the macro perspective. In view of the abovementioned problems, this paper selects the expressway trunk line as the main research area from a macro point of view and introduces the traffic flow prediction into the control strategy before the implementation, so as to predict the future traffic changes.

Therefore, this paper studies the combination of traffic volume prediction and control of expressway trunk line with VSL strategy. The contributions of this paper are as follows: (1) this paper simulates the overall situation of vehicles on the road based on the macroscopic traffic flow model and gives the control strategy from the macro level, which fills up the gaps in related research and (2) generates real-time dynamic demand that can be loaded on the transportation network through traffic estimation and forecasting technology. The dynamic road network traffic demand can more realistically verify the effect of the control strategy. (3) An optimal speed control strategy for highway arterials is proposed, based on optimization models and design 
algorithms. (4) The feasibility of the proposed model is verified by traffic simulation technology.

\section{Traffic Demand Estimation and Prediction}

Based on the prediction of traffic demand, the real-time traffic demand is obtained. Also, the traffic demand can be obtained by performing OD prediction function. The decision about the boundary of the queue length should be made in advance. Thus, the estimated or predicted traffic demand will be loaded on traffic network, and then the traffic state can be acquired to realize active management for dynamic traffic.

The key to real-time dynamic traffic demand forecasting is to obtain the OD amount in the future time period based on the historical time-varying OD matrix. Okutani [29] first established a state space model for dynamic OD estimation and verified the effect of the model through Kalman filtering. Kachroo [30] studied the applicability of the Kalman filter method in estimating the origin-destination of travelers within the network from the link traffic. On this basis, Ashok [31] made further corrections and analyzed the deviation between the predicted OD value and the historical value. In this paper, the state space model of Ashok is used as the estimation and prediction of real-time OD matrix. Using deviation to define state variable, $\delta x_{h}=x_{h}-x_{h}^{H}$, clearly, $x_{h}$ is the OD vector. The corresponding historical estimate is $x_{h}^{H}$. The deviation shows an autoregressive process:

$$
x_{h+1}=\sum_{p=h+1-q^{\prime}}^{h} f_{h+1}^{p} \delta x_{p}+w_{h} .
$$

As an autoregressive coefficient matrix, $f_{h+1}^{p}$ is the impact of $\delta x_{p}$ on $\delta x_{h+1}$; qis the autoregressive process times; $w_{h+1}$ is the white Gaussian noise. Satisfying $E\left(w_{h+1}\right)=0, E\left(w_{h} w_{l}^{T}\right)=Q_{h} \delta_{h l}$, the error covariance matrix is $Q_{h}, \delta_{W}= \begin{cases}1, & h=1, \\ 0, & h \neq 1 .\end{cases}$

$$
\delta y_{h}=\sum_{p=h-p^{\prime}}^{h} a_{h}^{p} \delta x_{p}+v_{h} .
$$

In the formula, $\delta y_{h}=y_{h}-h_{h}^{H}=y_{h}-\sum_{p-h-p^{\prime}}^{h} a_{h}^{p} x_{p}^{H}$. $y_{h}$ means the volume that is obtained during the interval of $h$, $y_{h}^{H}$ serves as the historical values. $a_{h}^{p}$ is an assignment dynamic OD matrix, which is the contribution of $\delta x_{p}$ to $\delta y_{p}$. The fraction of $r$ in OD departs from the original place during the interval of $p$. Then, during the interval $h$, it crosses the counting point. $p^{\prime}+1$ stands for the maximum time intervals in travelling between the OD pairs of the traffic network. $v_{h}$ is the error measurement, $E\left(v_{h}\right)=0$. To achieve the estimation and prediction of the dynamic OD, equations (1) and (2) are employed for the construction of the state space model. Also, the Kalman filter [32] is helpful for solving this problem.

\section{TheImproved Control Model}

4.1. The Improved Traffic Flow Model Integrated with VSL. Generally, there are three types of traffic flow models: macro-, micro- and mesoscopic models. The macroscopic model focuses on the collect behaviour of vehicles, the speed and density are the research objects. The microscopic model aims to describe the behaviour of individual vehicle in detail. The description range of mesotraffic model is between micro and macro, which is suitable for medium-sized traffic network. In this paper, the macro hierarchical control model [33] will be explored and improved based on the characteristics of highway. At present, the research on variable speed control strategy is not thorough. Besides, the control models employed in these studies are only based on few control strategies. A comprehensive and effective variable speed decision system is not established yet. To develop an effective decision system, the VSL control strategy is introduced in the macro hierarchical traffic flow control model. The speed-density relation will change if the speed limits are added to the macro hierarchical control model. The definitions of indexes and variables are shown in Table 1.

The improved macromodel-integrated VSL is proposed as follows:

$$
\begin{aligned}
p(i, k+1) & =p(i, k)+\frac{T}{l_{1} \bullet n_{i}}[q(i-1, k)-q(i, k)-e(i, k)], \\
q(i, k) & =p(i, k) \cdot \min \left\{v(i, k), v_{\lim }(i, k)\right\} \cdot n_{i} \\
v[p(i, k)] & =\min \left\{v_{f}\left[1-\left(\frac{p(i, k)}{p_{\mathrm{jam}}}\right)^{\alpha}\right]^{\beta}, v_{\mathrm{lim}}(i, k)\right\}
\end{aligned}
$$

At the entrance and exit of the boundary ramp, namely, $i=1$ and $i=N$,

$$
\begin{aligned}
p(1, k+1) & =p(1, k), \\
v(1, k+1) & =\min \left\{v[p(1, k)], v_{\lim }(1, k+1)\right\}, \\
p(N, k+1) & =p(N, k), \\
v(N, k+1) & =\min \left\{v\left[p(N, k), v_{\lim }(N, k+1)\right]\right\} .
\end{aligned}
$$

The queue length of the on-ramp is as follows:

$$
p(i, k+1)=p(i, k)+T[d(i, k)-r(i, k)] .
$$

In addition to the state equation $(6), r(i, k)$ must meet the following constraints:

$$
r_{i \min } \leq r(i, k) \leq r_{i \max } .
$$

So, $V=\left\{v_{\lim }(i, j), \quad i=1,2, \ldots ; j=1,2, \ldots\right\}$, is the variable speed limits strategy.

The relationship between density and speed on a segment can be described by equation (4) (see Figure 1).

In addition to the presented model, the dynamic characteristics of the vehicles are described. In this paper, the control objective is to minimize the total delay and the total travel time of all vehicles. This is a global optimal control algorithm on the basis of the mentioned state equations of the traffic flow model.

4.2. The Optimization Control Model. The multiobjective optimization control model, in this paper, aims to minimize 
TABLE 1: Table of definitions of variables.

\begin{tabular}{|c|c|}
\hline Variables & The definitions of variables \\
\hline$i$ & $i=1, \ldots, N$ \\
\hline$k$ & A period of time, $k=1,2, \ldots, k$ \\
\hline$l_{i}$ & It is the length of segment $i$ \\
\hline$n_{i}$ & It is the number of lanes on segment $i$ \\
\hline$T$ & The sampling period \\
\hline$v_{f}$ & The free flow speed \\
\hline$\rho_{\text {jam }}$ & The traffic jam density \\
\hline$\rho_{c}$ & The critical density \\
\hline$\alpha, \beta$ & Model parameters \\
\hline$\rho(i, k)$ & $\begin{array}{c}\text { At time } k \text {, on segment } i \text {, the vehicle density } \\
\text { (veh } / \mathrm{km} / \text { Lane) }\end{array}$ \\
\hline$r(i, k$ & At time $k$, on segment $i$, the entrance ramp traffic flow \\
\hline$e(i, k)$ & At time $k$, on segmen \\
\hline$v(i, k)$ & $\begin{array}{l}\text { At time } k \text {, on segment } i \text {, the average speed of vehicles } \\
\qquad(\mathrm{km} / \mathrm{h})\end{array}$ \\
\hline$p(i$, & At time $k$, on segment $i$, the queue length \\
\hline$d(i$, & At time $k$, on segment $i$, the traffic demand \\
\hline$q(i, k)$ & $\begin{array}{l}\text { At time } k \text {, on segment } i \text {, the number of vehicles } \\
\text { (veh/h/lane) }\end{array}$ \\
\hline$v_{\lim }(i, k)$ & At time $k$, on segment $i$, the limited speed \\
\hline
\end{tabular}

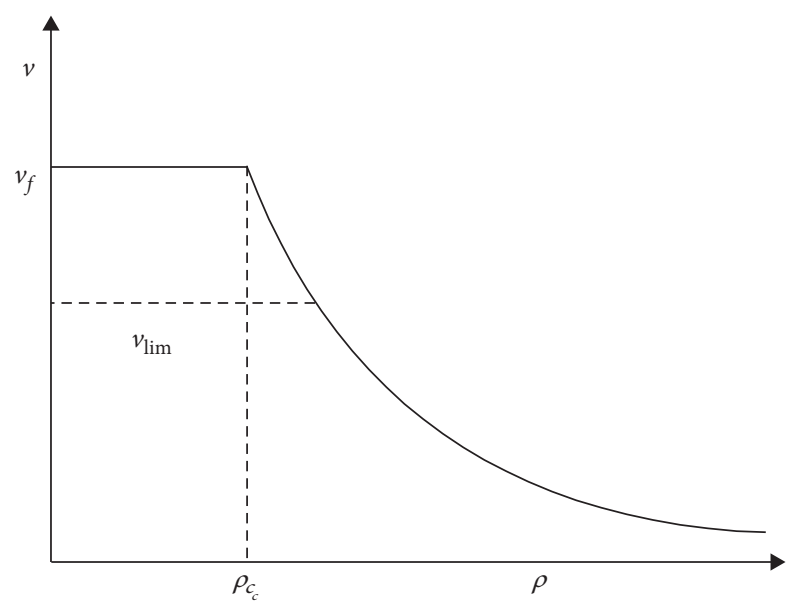

Figure 1: Speed and density relationship.

the total delay and the total travel time of all vehicles of all simulation intervals through the network. In other words, the objective is to ensure all vehicles reach destinations in the shortest possible time. One of the best variable speed limit strategies can be selected by means of simulation method. The following notations are used to provide a clear description of the problem.

The traffic network consists of nodes and connecting lines. The lines are divided into segments, which are represented by $j$. The total simulation time is divided into several intervals, which are represented by $h, Q(j, h)$ is the average traffic flow in hinterval on jsegment, $V(j, h)$ is the average traffic speed in hinterval on jsegment, and $p(j, h)$ is the average traffic density in hinterval on jsegment. Equation (8), formula of the objective function, reads

$$
\begin{aligned}
& \min L(\cdot)=\sum_{j h}\left(C_{j}-Q(j, h)+V(j, h)\right), \\
& \text { s.t. } C_{\min } \leq C \leq C_{\max }, \\
& Q(j, h), V(j, h), \rho(j, h) \geq 0,
\end{aligned}
$$

where $C_{\min }$ is the minimum traffic capacity and $C_{\max }$ is the maximum capacity. The constraints are up threshold and nonnegative.

\section{The Simulation Method}

The feasibility of online macroscopic traffic simulation and optimal traffic control is demonstrated in this paper. This approach can adapt to the changeable traffic conditions. A situation is proposed in which the VSL can be used in upstream main roads. The following is how the approach performs its function. The VSL can prevent traffic congestion at the bottleneck by creating a free flow zone at the upstream section. The existence of VSL changes the fundamental diagram shown in Figure 1and reduces the output capability of this segment.

The following section shows the process of executing the proper simulation with VSL. A closed cycle scheme plays an important part in solving the problem (see Figure 2). VSL is integrated into the mesoscopic traffic simulator to estimate network operation state. The optimal objective is the optimal control speed limits strategy by minimizing the total delay. In order to improve the estimation accuracy of macroscopic model parameters (such as $\alpha, \beta, k_{\text {jam }}$ etc. ), it has a process of parameter correction. A point deserves noting that the parameters usually become constant when the parameter calibration horizon passes. The procedure reduces the number of variables and enhances the stability of the traffic system. The parameters calibration process is chosen with the purpose of correcting system parameters. In this way, the network description and traffic dynamics characteristics can be obtained more accurately. In the following, a new optimization's performing functions are triggered to find the best strategy. In this regard, traffic estimation and prediction are of great help to this. A more comprehensive network state results from their interaction. Known as rolling horizon, this scheme enables updating the state from measurement and it can even update the model in every iteration step.

\section{The Simulation Study}

6.1. The Simulation Environment. As shown in the simulation structure diagram in Figure 3, the highway network is divided into 9 sections. Each section is a one-way four-lane connecting line. On this highway, there are 4 entry ramps and 4 exit ramps. The section of each road is $3 \mathrm{~km}$.

The proposed model is used in the modeling and simulation. In order to simulate the real traffic situation and reveal its essence, the parameters of the traffic flow model are set according to the specific characteristics of the road section (length, width, level, capacity, and traffic flow), where $\alpha=1.7, \beta=2, r_{\max }=900 \mathrm{veh} / \mathrm{h}, r_{\min }=180 \mathrm{veh} / \mathrm{h}$, $v_{f}=100 \mathrm{~km} / \mathrm{h}, \quad p_{\text {jam }}=130(\mathrm{veh} / \mathrm{km}) /$ Lane, and the 


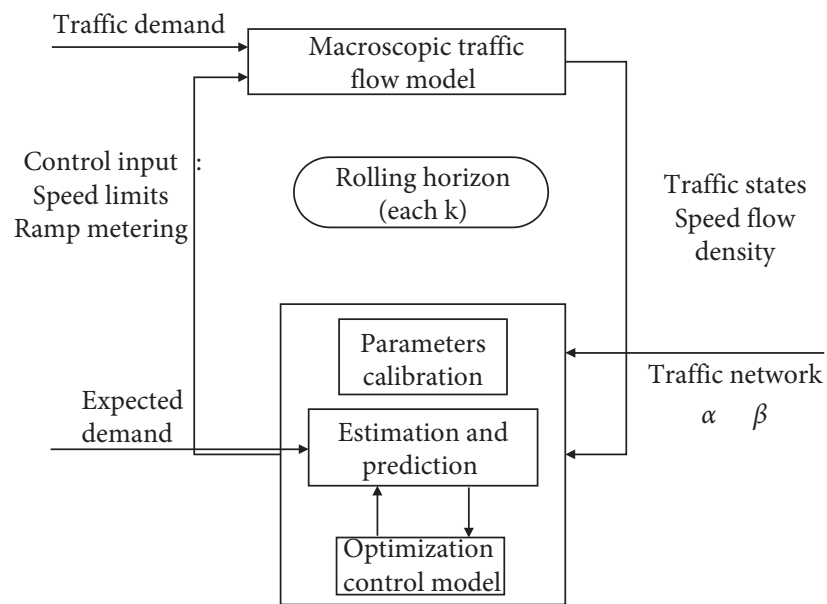

FIgURE 2: Simulation structure.

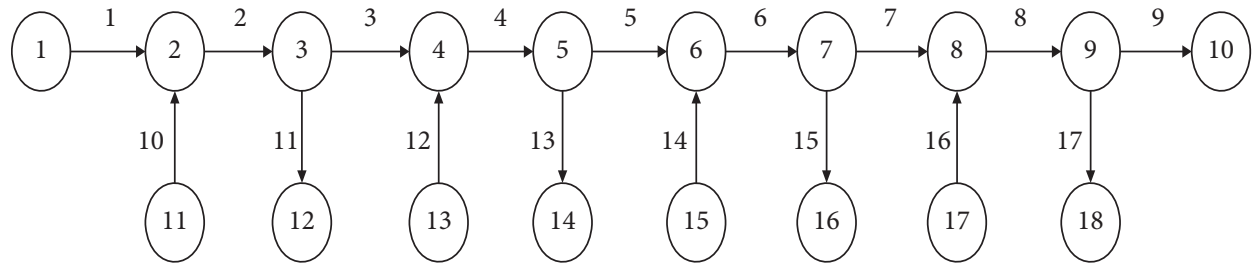

FIGURE 3: Simulation structure.

TABLE 2: The OD pairs in network.

\begin{tabular}{lcc}
\hline OD & Origin-destination & Average exit rate \\
\hline 1 & $1-12$ & 60 \\
2 & $1-14$ & 100 \\
3 & $1-16$ & 250 \\
4 & $1-18$ & 420 \\
5 & $1-10$ & 1500 \\
6 & $11-12$ & 50 \\
7 & $11-14$ & 300 \\
8 & $11-16$ & 360 \\
9 & $11-18$ & 220 \\
10 & $11-10$ & 300 \\
11 & $13-14$ & 40 \\
12 & $13-16$ & 120 \\
13 & $13-18$ & 840 \\
14 & $13-10$ & 420 \\
15 & $15-16$ & 60 \\
16 & $15-18$ & 100 \\
17 & $15-10$ & 500 \\
18 & $17-18$ & 80 \\
19 & $17-10$ & 360 \\
\hline
\end{tabular}

simulation time is 3 hours. The OD and exit average rate are shown in Table 2 . The average exit rate is a time variable, which reflects the average starting rate between OD pairs. A greater value indicates that the adjacent vehicle's departure time interval is shorter. In the simulation, we placed three variable message boards on road sections numbered 3,5 , and 7. the VSL strategy is as follows: $\{70,60,50\} ;\{60,50,50\}$. In the simulation time of 15-25 minutes and 35-45 minutes,
TABLE 3: Dynamic OD prediction result.

\begin{tabular}{|c|c|c|c|c|c|c|c|c|c|c|}
\hline \multirow{2}{*}{$\mathrm{O}$} & \multicolumn{10}{|c|}{$\mathrm{D}$} \\
\hline & 1 & 2 & 3 & 4 & 5 & 6 & 7 & 8 & 9 & 10 \\
\hline 1 & & & 21.00 & & 20.00 & & 56.00 & & 52.00 & 198.00 \\
\hline 2 & & & 5.00 & & 10.00 & & 26.00 & & 33.00 & 60.00 \\
\hline 3 & & & & & 21.00 & & 14.00 & & 100.00 & 72.00 \\
\hline 4 & & & & & & & & & & \\
\hline 5 & & & & & 3.00 & & 48.00 & & 55.00 & 102.00 \\
\hline 6 & & & & & & & & & & \\
\hline 7 & & & & & & & 7.00 & & 17.00 & 36.00 \\
\hline 8 & & & & & & & & & & \\
\hline 9 & & & & & & & & & & 30.00 \\
\hline 10 & & & & & & & & & & \\
\hline
\end{tabular}

the limit strategies are implemented. Vehicle detectors are installed at the middle of each section, so as to mark the traffic volume and average speed and density on the segment.

6.2. The Simulation Results. According to the traffic demand estimation and forecasting method, the dynamic traffic demand of the study area can be obtained. The prediction results are shown in Table 3 . After completing the traffic demand forecast, we first distribute the forecast results to the road network in the study area to obtain the traffic flow status and traffic flow parameters of each road section. Then, on this basis, the changes of traffic flow parameters before and after the implementation of the VSL strategy under the 
TABLE 4: Simulation results.

\begin{tabular}{lcc}
\hline Internal & The network average density without VSL & The network average density with VSL \\
\hline 1 & 0.00288 & 0.00285 \\
2 & 0.00681 & 0.00688 \\
3 & 0.00793 & 0.00804 \\
4 & 0.00845 & 0.00859 \\
5 & 0.00843 & 0.00839 \\
6 & 0.00847 & 0.00853 \\
7 & 0.00830 & 0.00861 \\
8 & 0.00817 & 0.00866 \\
9 & 0.00835 & 0.00851 \\
10 & 0.00850 & 0.00864 \\
11 & 0.00854 & 0.00870 \\
Average & 0.00853 & 0.00863 \\
\hline
\end{tabular}

overall network are compared, and the effect of the model in this paper has been verified.

Table 4shows the traffic density of each road section in the network before and after the implementation of the VSL strategy. What is worth noting here is that the entire network is taken into consideration in obtaining the simulation results. Obviously, before the VSL comes into play, the overall average density of the network is 0.00778 . After it is performed, the value is 0.00792 , a slight increase in density indicates that a decrease in speed results in an increase in the number of vehicles stranded on the network. The average traffic and speed of road sections in the network before the implementation of VSL were 387.4769 and 20.7170. However, after the implementation of VSL, the average network traffic was 387.2130 and the average speed was 20.5129. Due to the implementation of the variable speed limit strategy, the speed of the vehicle in the network has become lower, so the driving time of the vehicle in the network has increased.

The following two-dimensional and three-dimensional distribution map, respectively, depicts the average density and speed of each segment in each time period. Figure 4 is the three-dimensional distribution of the average density on each segment in each interval without VSL. Figure 5is the three-dimensional distribution of the average density on each segment in each interval with VSL. Figures 6and 7are their two-dimensional representations.

According to Figures 4-7, the average density of segments which are implemented with the VSL strategy increases significantly. But overall the other segments' average density becomes smoother. Because of the proposed method with VSL strategy, the simulation results present a better queue formation than those without a non-VSL. Owing to the VSL, it is convenient for vehicles to keep the earlier speed, because they can adjust speed according to the network state. In this way, the queue can be transferred from the bottleneck to upstream timely. However, the queue without the help of VSL cannot achieve that goal. Although after using VSL, the mean speed, especially during the rush hour, of most sections is lower than that in the non-VSL case, there is no negative impact on travel time. This highlights the advantages of the VSL in enabling the network to operate at a more stable speed. A comparison of the average speed of each interval from the eighth to twelfth interval implies

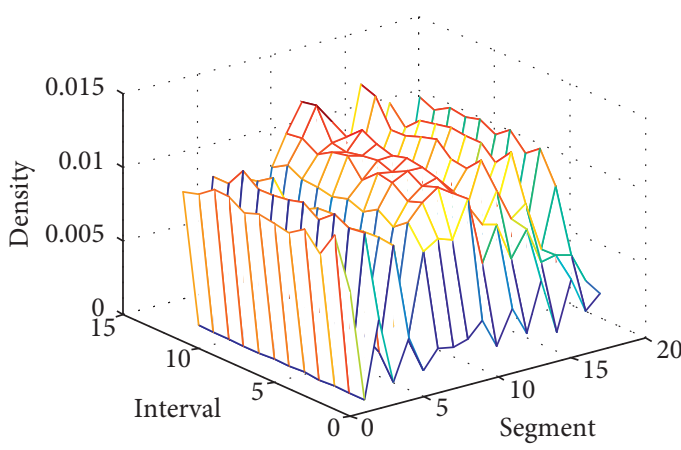

FIgURE 4: The density distribution without VSL.

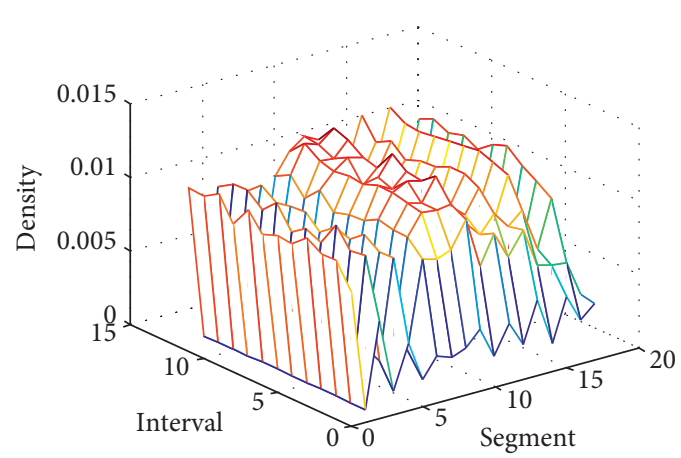

FIGURE 5: The density distribution with VSL.

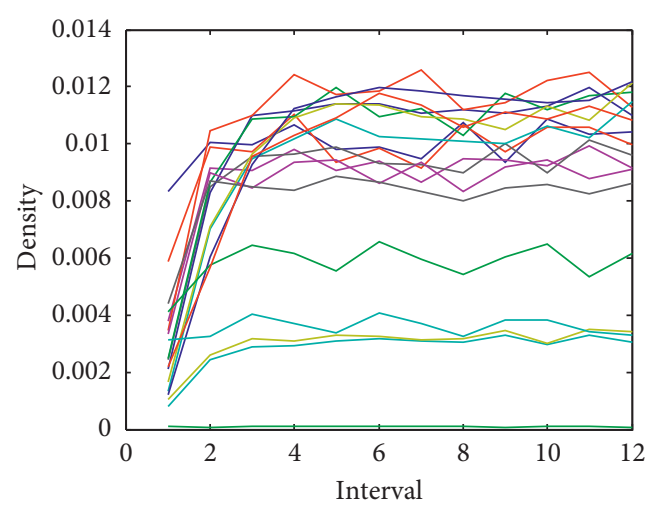

FIgure 6: Two-dimensional density without VSL. 


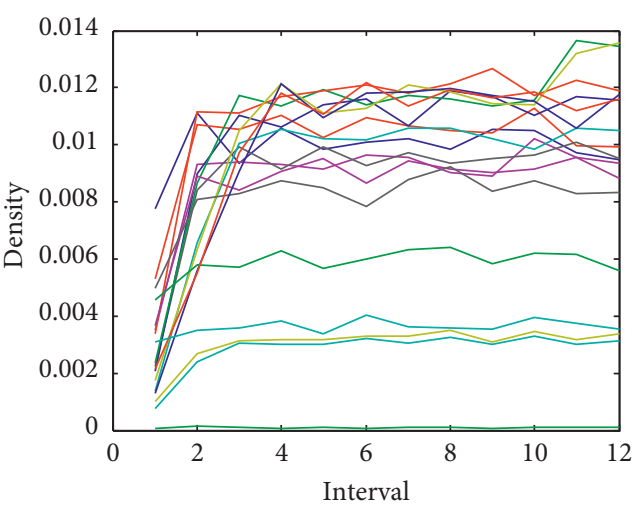

FIgUre 7: Two-dimensional density with VSL.

that the values are 17.7258, 16.7817, 16.9283, 18.6175, and 18.9500 , when there is no VSL. The values become, however, $17.8033,17.5525,17.0200,17.5867$, and 17.0967, when the VSL is used. The network of traffic flow becomes more stable than that in the previous time. The traffic flow of some segments becomes less congested. Theoretically, the more stable the traffic flow is, the better the operation efficiency of the traffic system will be.

In the same way, Figures $8-15$ show the average flow and speed of each segment in each interval in three-dimensional and two-dimensional forms.

In the four locations, the values of variable speed limit are set $100 \mathrm{~km} / \mathrm{h}, 80 \mathrm{~km} / \mathrm{h}, 80 \mathrm{~km} / \mathrm{h}$, and $90 \mathrm{~km} / \mathrm{h}$, respectively. Different values should be taken to be in line with limiting strategies. Because traffic congestion does not occur at those segments, variable speed limit strategies are acceptable in relieving traffic density while the overall traffic flow remains stable. When critical density appears on traffic network, the VSL plays an important role in avoiding potential danger. Both traffic density and traffic flow on the sections are far away from variable speed limit sections which undergo no changes. It can be concluded that without reducing road capacity, the variable speed limit strategies contribute to traffic safety.

Undoubtedly, if the vehicle speed is stable, it benefits to traffic flow operation. This avoids the risk of traffic accidents. To sum up, the variable speed limit strategies can effectively prevent the potential risk factors and improve the operation efficiency of the system in some cases. Although it does not show obvious effect in relieving traffic congestion, it is the best application in traffic management.

On the basis of the abovementioned research, in order to further verify the effectiveness of the method proposed in this article, we adopted a method of randomly perturbing the test data, that is, randomly changing each OD pair with a range of $30 \%$. Since the traffic demand of the expressway network is time-varying, adding random disturbances can better describe the dynamic changes of the traffic volume on the road network and then verifies the effectiveness of the method in this paper more realistically. The simulation results show that when the demand increases by $30 \%$ on individual road sections with high OD demand, the variable speed limit has little effect on the traffic flow of the road section. This is because the density of the road section

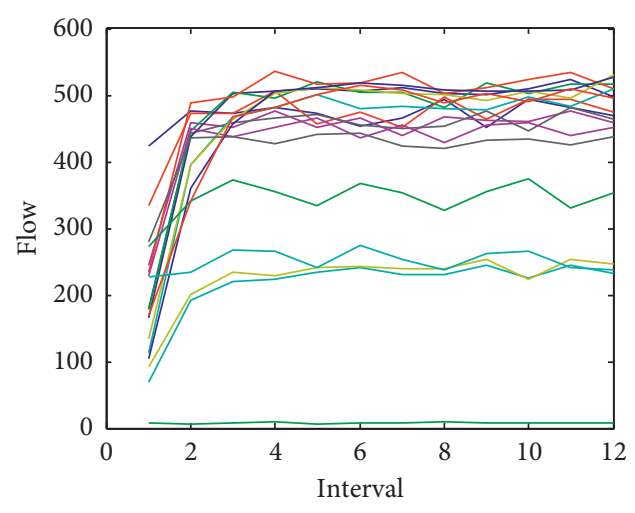

FIgURE 8: Two-dimensional flow without VSL.

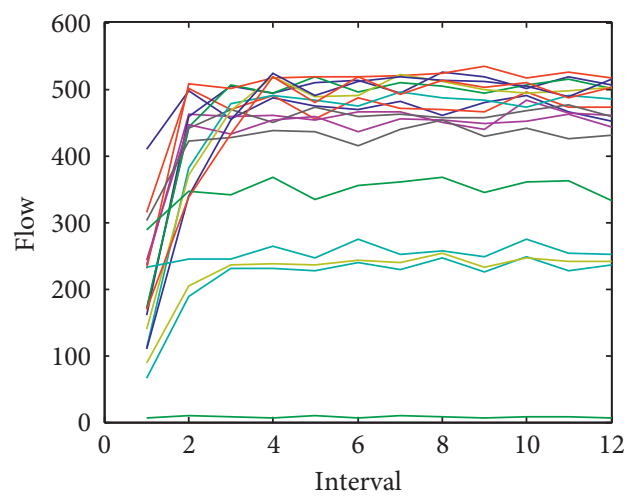

FIgURE 9: Two-dimensional density with VSL.

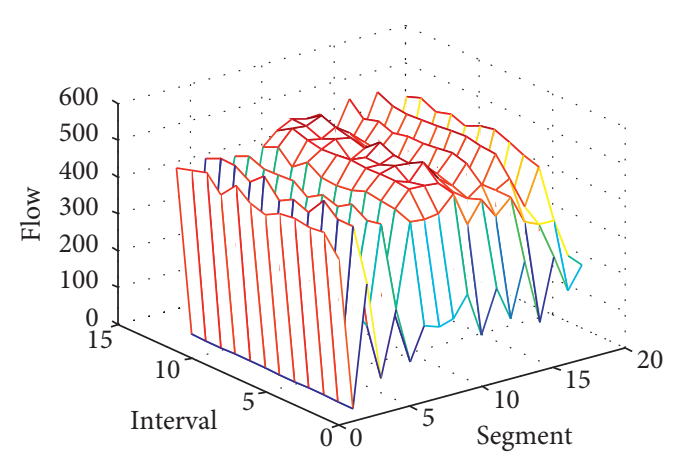

FIgure 10: The flow distribution without VSL.

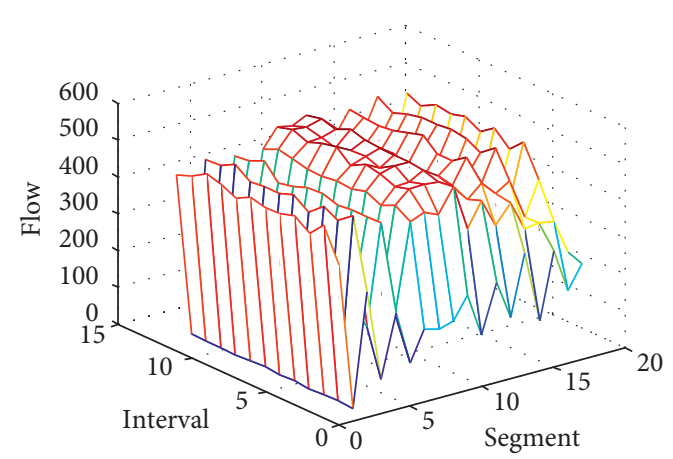

FIgURE 11: The flow distribution with VSL. 


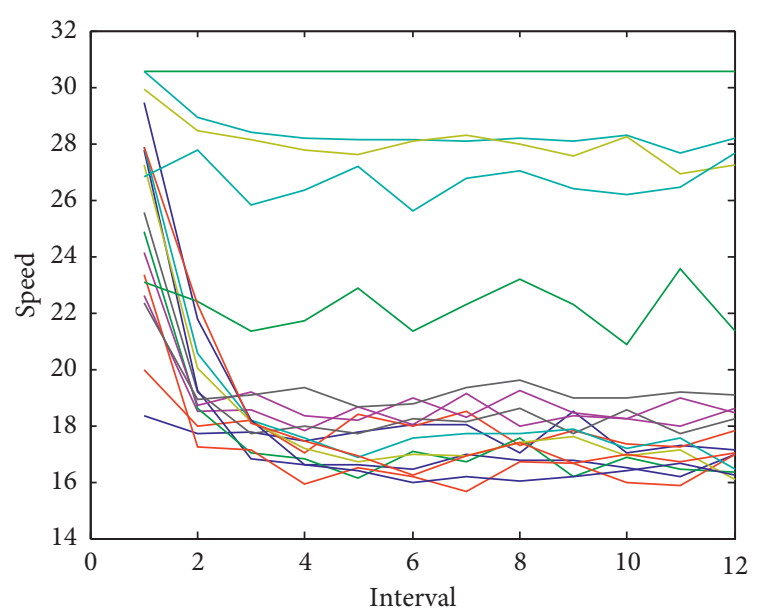

Figure 12: Two-dimensional speed without VSL.

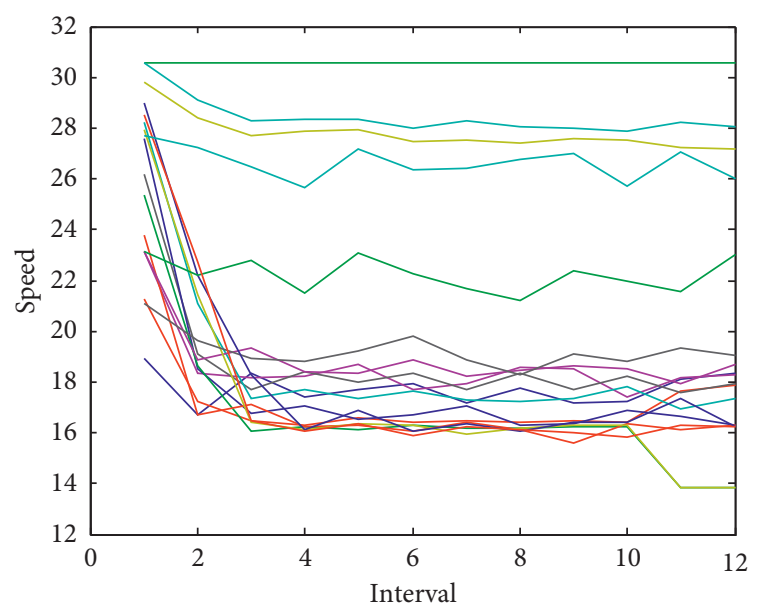

FIgUre 13: Two-dimensional speed with VSL.

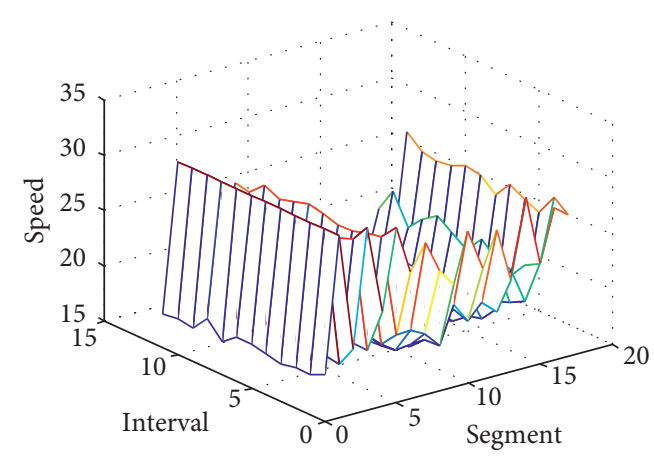

FIgURE 14: The speed distribution without VSL.

roughly makes the speed of the road section much lower than the dynamic speed limit value. When the demand is reduced by $30 \%$, the speed of the vehicles on the road section is greater than the speed limit value. At this time, the variable speed limit value can act on most vehicles on the road section, thus producing a more obvious improvement and

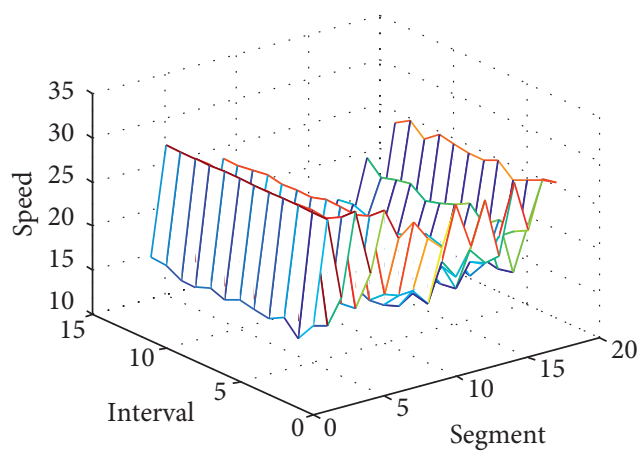

FIgUre 15: The speed distribution with VSL.

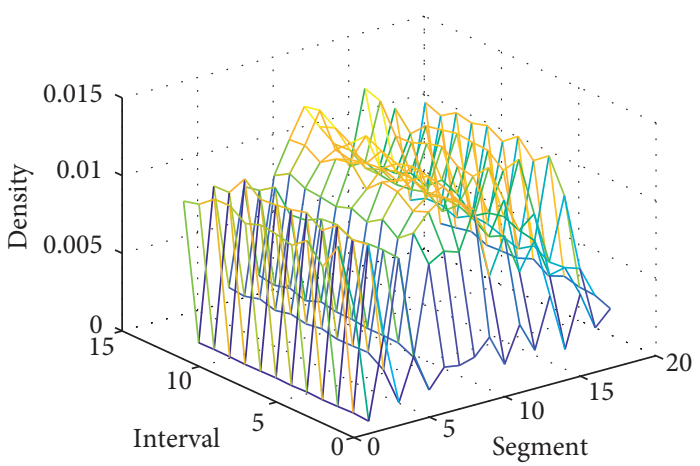

FIgURE 16: The VSL-integrated mesomodel.

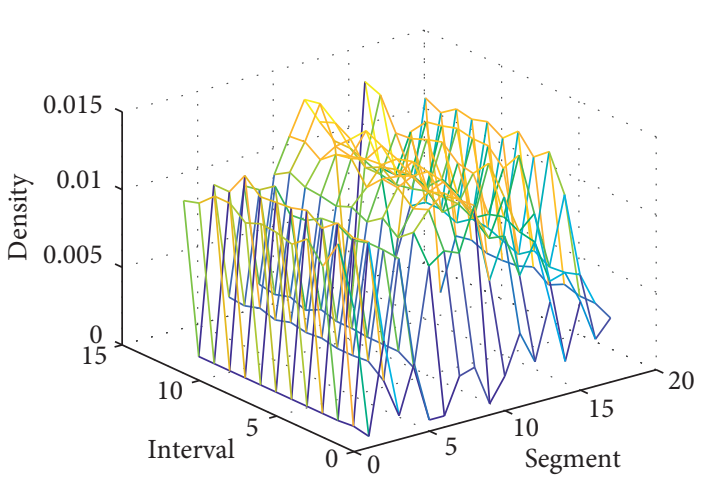

Figure 17: The VSL-integrated LTM.

regulation effect. In general, it can be concluded through simulation: on the one hand, the variable speed limit method proposed in this paper can increase the traffic in some road sections, and the density is evenly distributed in the overall road network. On the other hand, the model has a more obvious improvement effect before the occurrence of congestion, so the implementation of the strategy needs to cooperate with accurate traffic state prediction methods. In addition, if the OD is adjusted again, the control measures of closed ramp can be adopted, combined with the variable speed limit of this article to adjust the main line traffic flow, and better control effect is desirable. 


\section{Comparing with Other Research Results}

The proposed method in this paper is suitable for specific sections of the expressway. We used the assumed theoretical data to verify it. In this section, we compared the results of this paper with three other traffic flow models integrated with VSL, namely, the mesotraffic flow model, CTM (the cell transport model), and LTM (the link transport model) [14, $34,35]$. CTM is suitable for studying the micromotion of urban road vehicles and is combined with VSL to control the congestion at the intersection at the same time ensuring traffic safety. LTM is suitable for studying the traffic conditions at the section level which can nip the traffic congestion in the bud according to the speed limit strategy of different road sections. The mesoscopic traffic flow model integrated with VSL can control regional urban traffic. At the same traffic environment, we use VSL-integrated CTM and the VSL-integrated mesoscopic traffic flow model to study the traffic flow parameters such as density, and the results are shown in Figures 16and 17.

From the abovementioned results, there is little difference among the two types of models. The density calculated by the LTM-based model is a little higher than any one of others. Although the optimization effect of the traffic flow on the road is not very different, it is worth noting that the research scope of the expressway in this paper is very large, which shows that the calculation speed of the model in this paper is faster than other methods, so it is suitable for the macrotraffic flow based on VSL.

\section{Research Conclusions and Prospects}

This paper explores the VSL strategy on expressway by means of traffic simulation method, and the real-time traffic demands are estimated and predicted. With the help of motion equation, a cooperative systems extension is developed. Then, a new speed limits strategy is proposed by using the proposed method. The recommendations of speed are provided in advance for the vehicles at predefined points. Additionally, according to the actual speed of the vehicles, the location of releasing speed limits strategy is determined.

According to the simulation results, the proposed method produces some different queue formations under the VSL strategy. These vehicles adapt their earlier speed to guiding vehicle speed, which continuously produces a queue that moves upstream from the bottleneck. In the non-VSL case, however, the queue is stationary and the front of the queue grows upstream from the bottleneck, which reduces the capacity of bottleneck. The new control method can also smooth the density oscillation of traffic flow. Then, the traffic flow is in a stable state. In the cooperative VSL case, the mean speed of most road segments during the rush hour is lower than that in the non-VSL case. But the lower mean speed does not affect the total travel time. This provides a new way to control the traffic flow with a stable speed pattern. It can be concluded that the proposed VSL strategy could harmonize traffic flow, promote traffic efficiency, and then improve the regional traffic conditions.

\section{Data Availability}

The data of this study come from mesoscopic simulation software.

\section{Conflicts of Interest}

The authors declare that there are no conflicts of interest regarding the publication of this paper.

\section{Acknowledgments}

This work was supported by the National Natural Science Foundation of China (Grant nos. 71871130, 71471104, 71771019, and 71971125); University Science and Technology Program Funding Projects of Shandong Province (Grant no. J17KA211); Social Science Foundation of Shandong Province (Grant no.12BJJJ13); and Science and Technology Program Funding Projects of Shandong Police College (Grant no. YSKYB2005).

\section{References}

[1] Z. Z. Tian, "Modeling and implementation of an integrated ramp metering-diamond interchange control system," Journal of Transportation Systems Engineering and Information Technology, vol. 1, no. 7, pp. 61-72, 2007.

[2] X. R. Liang, Z. Y. Liu, J. M. Xu et al., "Design and simulation of coordinated ramp control system on expressway," Information and Control, vol. 3, no. 7, pp. 308-311, 2005.

[3] O. J. Chen, A Dynamic Traffic Control Model for Real Time Expressway Operations, Massachusetts Institute of Technology, Boston, MA, USA, 1996.

[4] Y. Wang, X. L. Ma, Z. B. Li, Y. Liu, M. Xu, and Y. Wang, "Profit distribution in collaborative multiple centers vehicle routing problem," Journal of Cleaner Production, vol. 144, pp. 203-219, 2017.

[5] Y. Wang, S. G. Peng, X. S. Zhou, M. Mahmoudi, and L. Zhen, "Green logistics location-routing problem with eco-packages," Transportation Research Part E: Logistics and Transportation Review, vol. 143, Article ID 102118, 2020.

[6] Y. Wang, Y. Y. Yuan, X. Guan et al., "Collaborative twoechelon multicenter vehicle routing optimization based on state-space-time network representation," Journal of Cleaner Production, vol. 258, Article ID 120590, 2020.

[7] P. W. Lin, K. P. Kang, and G. L. Chang, "Exploring the effectiveness of Variable Speed Limit controls on highway work-zone operations," Intelligent Transportation Systems, vol. 8, no. 3, pp. 155-168, 2004.

[8] A. Hegyi, B. D. Schutter, and J. Hellendoorn, "Optimal coordination of variable speed limits to suppress shock waves," IEEE Transportation on Intelligent Transportation System, vol. 1852, no. 1, pp. 167-174, 2003.

[9] R. L. Bertini, S. Boice, and K. Bogenberger, "Dynamics of a variable speed limit system surrounding a bottleneck on a German autobahn," in Proceedings of the 85th TRB Annual Meeting, Washington DC,WA, USA, January 2006.

[10] A. M. Abdel, J. Dilmore, and A. Dhindsa, "Evaluation of variable speed limits for real-time expressway safety improvement," Accident Analysis and Prevention, vol. 38, no. 7, pp. 335-345, 2006.

[11] H. D. Yao, J. X. Cui, X. P. Li, Y. Wang, and S. An, “A trajectory smoothing method at signalized intersection based on 
individualized variable speed limits with location optimization," Transportation Research Part D, vol. 62, 2018.

[12] F. G. Ellen and T. Andreas, "Using connected vehicles in a variable speed limit system," Transportation Research Procedia, vol. 27, pp. 85-92, 2017.

[13] M. D. Robinson, "Examples of variable speed limit applications. Speed management workshop," in Proceedings of the 79th TRB Annual Meeting, Washington DC, WA, USA, January 2000.

[14] S. B. Li and D. N. Cao, "Variable speed limit strategies' analysis with cell transmission model on expressway," Modern Physics Letters B, vol. 31, no. 24, Article ID 1750219, 2017.

[15] S. B. Li and D. N. Cao, "Variable speed limit strategies analysis with link transmission model on urban expressway," Modern Physics Letters B, vol. 32, no. 6, Article ID 1850077, 2018.

[16] S. B. Li, D. N. Cao, W. X. Dang, T. Sun, and W. Dang, "Intersection-movement-based traffic incidents detected method and run state evaluation of expressway network," Journal of Transportation Safety and Security, vol. 11, no. 6, pp. 642-660, 2018.

[17] A. M. Abdel, R. Cunningham, V. Gayah, and L. Hsia, "Dynamic variable speed limit strategies for real-time crash risk reduction on freeways," Transportation Research Record Journal of the Transportation Research Board, vol. 2078, no. 2078, pp. 108-116, 2009.

[18] E. Ivanjko, K. Kušić, and M. Gregurić, "Simulational analysis of two controllers for variable speed limit control," Proceedings of the Institution of Civil Engineers-Transport, 2020.

[19] J. Fang, H. X. Ye, and S. M. Easa, "Modified Traffic Flow Model with Connected Vehicle Microscopic Data for Proactive Variable Speed Limit Control," Journal of Advanced Transportation, vol. 2019, Article ID 8151582, 11 pages, 2019.

[20] F. Alasiri, Y. H. Zhang, and A. I. Petros, "Robust variable speed limit control with respect to uncertainties," European Journal of Control, vol. 59, pp. 216-226, 2021.

[21] Q. Xu, L. H. Li, Z. W. Yi, P. Mao, and M. Yang, "Traffic flow modeling of freeway variable speed limit control based on the big data of driving behavior," Journal of Advanced Transportation, vol. 2020, Article ID 8859494, 11 pages, 2020.

[22] S. B. Li, J. J. Wu, Z. Y. Gao et al., "Bi-dynamic analysis of traffic congestion and propagation based on complex network," Acta Physica Sinica, vol. 60, no. 11, Article ID 050701, 2011.

[23] J. C. Long, Z. Y. Gao, and P. Orenstein, "Control strategies for dispersing incident-based traffic jams in two-way grid networks," IEEE Transactions on Intelligent Transportation Systems, vol. 13, no. 2, pp. 469-481, 2012.

[24] A. M. H. Zhang, Z. Y. Gao, and H. L. Ren, "Incident-based traffic congestion control strategy," Science China Technological Sciences, vol. 54, no. 5, pp. 1338-1344, 2011.

[25] H. W. Guo, W. H. Wang, W. W. Guo, X. Jiang, and H. Bubb, "Reliability analysis of pedestrian safety crossing in urban traffic environment," Safety Science, vol. 50, no. 4, pp. 968-973, 2012.

[26] W. H. Wang, W. Zhang, H. W. Guo, H. Bubb, and K. Ikeuchi, "A behavioural car-following safety model for microscopic simulation of traffic flow with various driving characteristics," Transportation Research Part C, vol. 19, pp. 1202-1214, 2011.

[27] J. J. Tang, F. Liu, Y. J. Zou, W. Zhang, and Y. Wang, “An improved fuzzy neural network for traffic speed prediction considering periodic characteristic," IEEE Transaction On Intelligent Transportation Systems, vol. 18, no. 9, pp. 23402350, 2017.

[28] J. J. Tang, J. Zeng, Y. W. Wang, H. Yuan, F. Liu, and H. Huang, "Traffic flow prediction on urban road network based on license plate recognition data: combining attention-LSTM with genetic algorithm," Transportmetrica A: Transport Science, vol. 17, no. 4, pp. 1217-1243, 2021.

[29] I. Okutani and J. Y. Stephanedes, "Dynamic prediction of traffic volume through Kalman filtering theory," Transportation Research Part B: Methodological, vol. 18, no. 1, pp. 1-11, 1984.

[30] P. Kachroo, K. Ozbay, and A. Narayanan, "Investigating the use of Kalman filtering approaches for dynamic origin-destination trip table estimation," Electrical and Computer Engineering, vol. 1, pp. 138-142, 1997.

[31] K. Ashok and A. M. Ben, "Dynamic origin-destination matrix estimation and prediction for real-time traffic management systems," in Proceedings of 12th International Symposium on Transportation and Traffic Theory, Amsterdam Elsevier Science, Berkly, CA, USA, July 1993.

[32] Y. S. Suh, V. H. Nguyen, and Y. S. Ro, "Modified Kalman filter for networked monitoring systems employing a send-on-delta method," Automatica, vol. 43, no. 2, pp. 332-338, 2007.

[33] Z. Y. Liu, Theory and Application of Intelligent Traffic Control, Science Press, Beijing, China, 2003.

[34] S. B. Li, D. N. Cao, W. X. Dang, and L. Zhang, "Variable speed limit strategies analysis with mesoscopic traffic flow model based on complex networks," International Journal of Modern Physics C, vol. 29, no. 2, Article ID 1850014, 2018.

[35] S. B. Li, T. Wang, H. L. Ren, X. Kong, and X. Wang, "Coordination optimization of VSL strategy on urban expressway and main road intersection signal," IEEE Access, vol. 8, pp. 223976-223987, 2020. 